\title{
Flexible metamaterials for wireless strain sensing
}

Rohat Melik, Emre Unal, Nihan Kosku Perkgoz, Christian Puttlitz, and Hilmi Volkan Demir

Citation: Appl. Phys. Lett. 95, 181105 (2009);

View online: https://doi.org/10.1063/1.3250175

View Table of Contents: http://aip.scitation.org/toc/apl/95/18

Published by the American Institute of Physics

\section{Articles you may be interested in}

Metamaterial-based wireless strain sensors

Applied Physics Letters 95, 011106 (2009); 10.1063/1.3162336

Flexible metasurfaces and metamaterials: A review of materials and fabrication processes at micro- and nano-scales

Applied Physics Reviews 2, 011303 (2015); 10.1063/1.4913751

Ultrasensitive terahertz sensing with high-Q Fano resonances in metasurfaces

Applied Physics Letters 105, 171101 (2014); 10.1063/1.4895595

Metamaterial near-field sensor for deep-subwavelength thickness measurements and sensitive refractometry in the terahertz frequency range

Applied Physics Letters 100, 221101 (2012); 10.1063/1.4722801

All-dielectric phase-change reconfigurable metasurface

Applied Physics Letters 109, 051103 (2016); 10.1063/1.4959272

Structural tunability in metamaterials

Applied Physics Letters 95, 084105 (2009); 10.1063/1.3211920

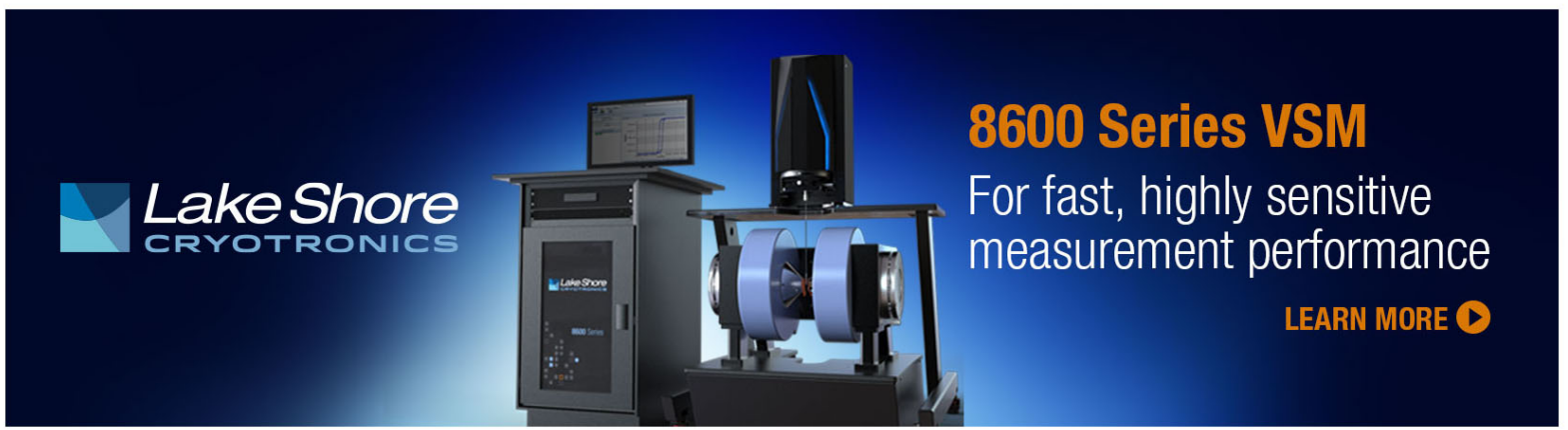




\title{
Flexible metamaterials for wireless strain sensing
}

\author{
Rohat Melik, ${ }^{1}$ Emre Unal, ${ }^{1}$ Nihan Kosku Perkgoz, ${ }^{1}$ Christian Puttlitz, ${ }^{2}$ and \\ Hilmi Volkan Demir ${ }^{1, a)}$ \\ ${ }^{1}$ Department of Electrical Engineering, Department of Physics, Nanotechnology Research Center, \\ and Institute of Materials Science and Nanotechnology, Bilkent University, Ankara 06800, Turkey \\ ${ }^{2}$ Department of Mechanical Engineering and School of Biomedical Engineering, Colorado State University, \\ Fort Collins, Colorado 80523, USA
}

(Received 9 July 2009; accepted 27 September 2009; published online 4 November 2009)

\begin{abstract}
We proposed and demonstrated flexible metamaterial-based wireless strain sensors that include arrays of split ring resonators (SRRs) to telemetrically measure strain. For these metamaterial sensors, we showed that a flexible substrate (e.g., Kapton tape) delivers greater sensitivity and a more linear response as compared to using silicon substrates. Specifically, these tape-based flexible SRR sensors exhibit a significantly improved sensitivity level of $0.292 \mathrm{MHz} / \mathrm{kgf}$ with a substantially reduced nonlinearity error of $3 \%$ for externally applied mechanical loads up to $250 \mathrm{kgf}$. These data represent a sixfold increase in sensitivity and a 16-fold reduction in error percentage. (c) 2009 American Institute of Physics. [doi:10.1063/1.3250175]
\end{abstract}

Telemetric strain measurement is important in many fields including civil engineering (e.g., to assess the strength of various concrete surfaces ${ }^{1}$ ) and the health sciences (e.g., to observe the healing process of fractures in bones ${ }^{2,3}$ ). Many applications require that these measurements occur on curved or nonplanar surfaces. To address these demands, we propose and demonstrate flexible metamaterial-based wireless strain sensors that telemetrically monitor strain in real time. The operating principle of these sensors relies on the shift of their operating frequency $\left(f_{0}\right)$ with an externally applied load to read out the strain remotely from the frequency shift. From a feasibility viewpoint, wireless sensors that operate on this principle are required to have their resonance frequency to be easily measureable, exhibiting relatively high quality factors ( $Q$-factors) with relatively large dips on resonance in their transmission spectra and being highly sensitive to the mechanical deformation with low nonlinearityerrors.

For remote sensing, metamaterial based architectures provide the ability to achieve higher $Q$-factors and larger resonance dips in transmission, compared to conventional radio frequency (rf) structures, as demonstrated in our previous works with silicon-based metamaterial strain sensors. ${ }^{4,5}$ But, for enhanced sensitivity and linearity, these metamaterial sensors further need to be mechanically flexible. In this paper, we designed, fabricated, and characterized flexible metamaterials for wireless strain sensing and demonstrated substantially increased sensitivity and significantly decreased nonlinearity errors, compared to our previously reported works. $^{4,5}$

There are many previous reports with respect to the use of metamaterials in various applications including negative refraction indices, ${ }^{6,7}$ focusing light, ${ }^{8}$ making superlenses, ${ }^{9}$ and cloaking. ${ }^{10}$ We have also previously used metamaterials in the fabrication of silicon wireless strain sensors in a double split ring resonator (SRR) architecture ${ }^{4}$ and in multiple nested SRR architecture. ${ }^{5}$ In this paper, different from

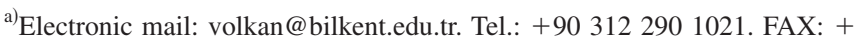
903122901015 .
}

the previous works of our group, this work introduces flexible metamaterials that are designed and fabricated on Kapton tape. This is a polyimide tape, also known as vacuum tape, commonly used in fabrication and packaging, such as in metal deposition, wave soldering, lithography, powder coating, and insulating circuit boards, because it is heat resistant and has silicone adhesive on the back side that does not leave any residue when the tape is removed. ${ }^{11}$

The fabrication procedure of our Kapton-based flexible metamaterial is depicted in Fig. 1(a). We first deposit $0.1 \mu \mathrm{m}$ thick $\mathrm{Au}$ on the tape using standard metallization techniques. Using plasma enhanced chemical vapor deposi-

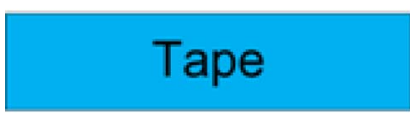

(i)

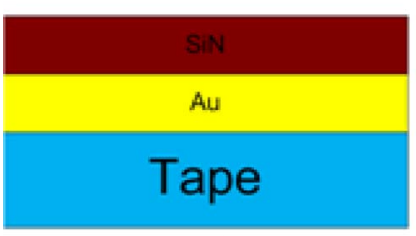

(iii)

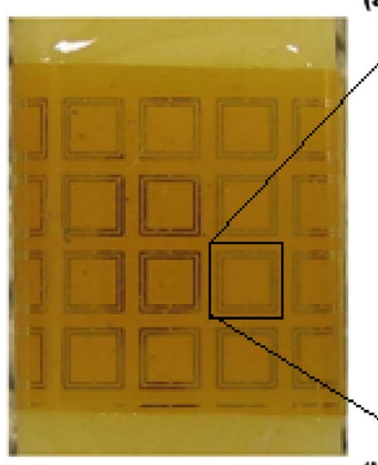

(b)

(a)

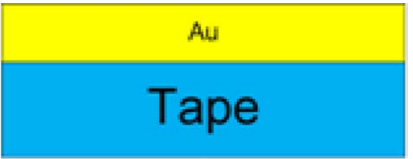

(盲)

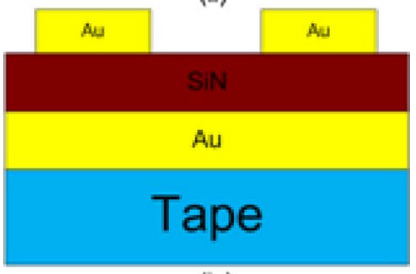

(iv)

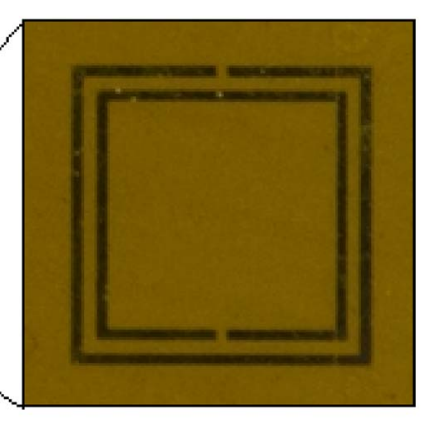

FIG. 1. (Color online) (a) Fabrication procedure of the tape-based flexible sensor and (b) the final fabricated structure of the tape-based flexible sensor. 
tion (PECVD), we subsequently deposit $0.1 \mu \mathrm{m}$ thick $\mathrm{Si}_{3} \mathrm{~N}_{4}$ as a dielectric thin film. Finally, we apply standard lithography, metal evaporation, and lift-off techniques to deposit and pattern a $0.1 \mu \mathrm{m}$ thick Au layer as top strata and finalize our sensor fabrication. In our microfabrication process, we were able to incorporate the Kapton vacuum tape since it can withstand up to $260{ }^{\circ} \mathrm{C}$, which is sufficient in our case as our highest temperature process (dielectric deposition in PECVD) is performed at $250{ }^{\circ} \mathrm{C}$.

The main difference in the fabrication procedure between these tape-based flexible sensors and the silicon-based sensors is the deposition of the first gold layer onto the vacuum tape substrate. This bottom gold layer increases the absorption of the sensor at the resonance frequency, producing a large dip at the resonance frequency. However, with the silicon substrate, the silicon inherently increases the absorption, so there is no need to deposit this extra Au layer. Deposition of the first gold layer also guarantees the presence of a parallel plate capacitor (between the first and final gold layers) of the tape-based flexible sensor. In the case of the silicon-based sensor, the silicon is doped; hence, there is no need for this additional Au layer to establish a parallel plate capacitor. The manufacture of a parallel plate capacitor is important for the operation of the sensor because, under loading, this capacitance will change and produce a resonance frequency shift. ${ }^{4}$ The final fabricated flexible metamaterial sensor can be seen in Fig. 1(b). Our SRR geometry has a $2220 \mu \mathrm{m}$ outer length and a $1380 \mu \mathrm{m}$ inner length. The sensor also has a $140 \mu \mathrm{m}$ inner width and a $140 \mu \mathrm{m}$ outer width, with a $280 \mu \mathrm{m}$ inner spacing and a $280 \mu \mathrm{m}$ outer spacing. The unit cell length of our SRR architecture is $2780 \mu \mathrm{m}$. The total resonator has $5 \times 5$ unit cells, producing a $1.5 \times 1.5 \mathrm{~cm}^{2}$ chip size.

For the silicon-based sensor, we use a hard epoxy to fix the sensor to the test stick (made of cast polyamide), which is used as the loading fixture. However, for the tape-based flexible sensor, there is no need for an additional epoxy layer because the tape has its own adhesive (silicone based epoxy) on the back side. Thus, the sensor is affixed to the test material directly. The goal of the characterization is to observe the shift of the operating frequencies under different loading magnitudes. Therefore, by observing this frequency change, the strain of the test material is measured telemetrically. In the experimental setup, we use one excitation transmitter and one receiver antenna to measure the spectral response of the sensors. We look at the transmission spectra (in $S_{21}$ configuration) to observe the resonance behavior.

Figure 2(a) shows transmission characterization of the silicon-based metamaterial sensor (in decibels), which demonstrates a $-10 \mathrm{~dB}$ transmission minimum or greater for all levels of loading in our experiment. Here we assign $\Delta f_{0}$ as the frequency shift with respect to the case of no load and denote the applied force as $F$, with $\Delta f_{0}$ versus $F$ illustrated in Fig. 2(b). The no load operating frequency of the sensor is $12.783 \mathrm{GHz}$. The Young's modulus of the cast polyamide is 3.287 GPa, which is measured by using data obtained from wired strain gauges (Tokyo Sokki Kenkyujo Co., Ltd. Strain Gauges with a gauge factor of 2.1) and simple elasticity theory. Using this data, we obtain a $0.0487 \mathrm{MHz} / \mathrm{kgf}$ sensitivity, or correspondingly $2.303 \times 10^{-3} \mathrm{MHz} /$ microstrain sensitivity, as shown in Fig. 2(b). In Fig. 2(c), we obtain less than a 600 microstrain nonlinearity error, and this corresponds to less than a $50 \%$ nonlinearity-error. ${ }^{12}$
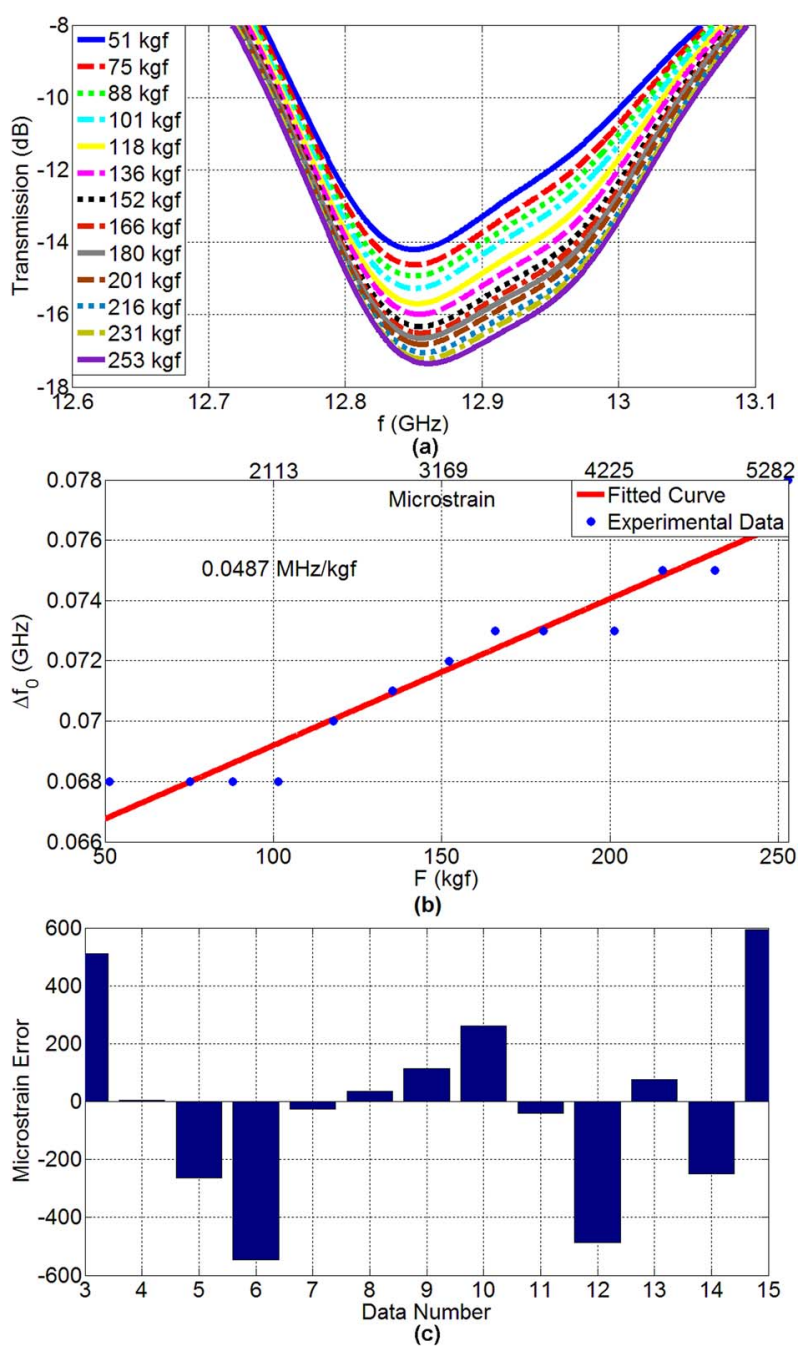

FIG. 2. (Color online) (a) Transmission spectra of the silicon-based sensor parameterized with respect to the external force, (b) its $F$ vs $\Delta f_{0}$ (and microstrain) graph, and (c) its nonlinearity errors in terms of microstrain.

Figure 3(a) depicts the relative transmission spectra (in decibels) of the tape-based flexible metamaterial sensor. The data indicate that the no load operating frequency is 12.208 $\mathrm{GHz}$ and a greater than $-10 \mathrm{~dB}$ relative minimum in the transmission spectra for all loading cases. Figure 3(b) demonstrates the applied load $(F \sim 30-250 \mathrm{kgf})$ versus $\Delta f_{0}$ (the frequency shift with respect to the no load case). The data indicate that the flexible sensor delivers a $0.292 \mathrm{MHz} / \mathrm{kgf}$ sensitivity corresponding to a $13.83 \times 10^{-3} \mathrm{MHz} /$ microstrain sensitivity. The data exhibit a less than $80 \mathrm{mi}-$ crostrain nonlinearity-error [as shown in Fig. 3(c)] with an associated 3\% nonlinearity-error. ${ }^{12}$ Thus, there is an appreciable increase in sensitivity (six times better) and a substantial decrease in nonlinearity-error percentage (16 times better) in the tape-based flexible metamaterial as compared to the silicon-based metamaterial.

The data show that the tape-based flexible metamaterial is more sensitive and more linear compared to the siliconbased metamaterial. When considering these results, one must take into account both the rf and mechanical aspects of the system. The silicon-based sensor delivers greater dip in transmission and higher $Q$ factors, and, hence delivers a higher signal-to-noise ratio as compared to tape-based flexible sensor. We postulate that this is because silicon is much 

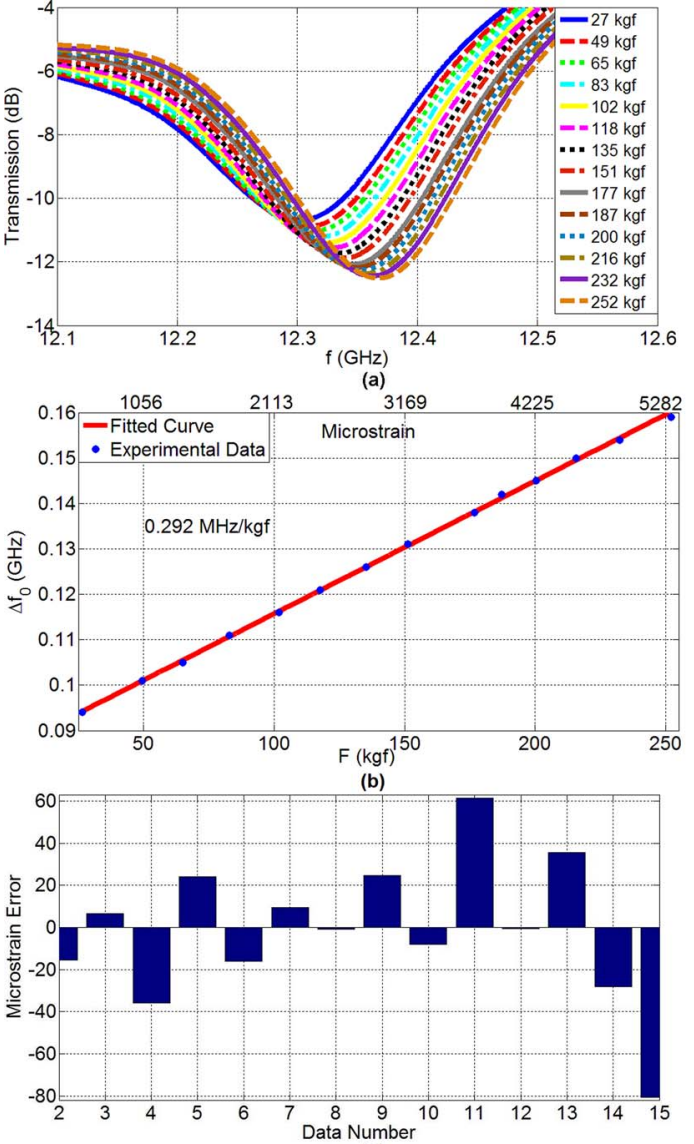

(c)

FIG. 3. (Color online) (a) Transmission spectra of the tape-based flexible sensor parameterized with respect to the external force, (b) its $F$ vs $\Delta f_{0}$ (and microstrain) graph, and (c) its nonlinearity errors in terms of microstrain.

thicker than the bottom gold layer on the vacuum tape, and, therefore, the silicon has a greater absorption at the resonance frequency. These factors account for the higher $Q$ factor obtained with the silicon sensor as compared to tapebased flexible sensor. Thus, by only considering rf portion of the system, it is not unexpected that the silicon-based sensor delivers a more linear response than the flexible metamaterial sensor. However, because of the mechanical aspect of the system, the sensor that incorporates the vacuum tape is more linear possibly because it uses a flexible substrate. Specifically, the flexible substrate has a lower elastic modulus and undergoes relatively greater mechanical deformation (for the same applied load), which results in a more sensitive construct. Since there are a limited number of datum points obtained in a single frequency scan by the network analyzer, it is easier to resolve smaller shifts in the transmission spectra in response to the externally applied load when the sensitivity is higher. If the network analyzer resolution is not sufficient to resolve the frequency shift with the applied load, then the resultant $F$ versus $\Delta f_{0}$ data becomes stepwise, which increases the nonlinearity errors, as is the case with the silicon-based metamaterial depicted in Fig. 2(b). The use of an external epoxy also plays an important role in the sensor's sensitivity and linearity. Since external epoxy is not required for fixation of the vacuum tape substrate to the test materials, the strain induced on the test materials directly propagates to the vacuum tape substrate. However, external epoxy is required to attach the silicon substrate to the test materials. Hence, part of the applied strain may not be directly conferred to the silicon substrate. We hypothesize that this rationale may explain why the silicon substrate's frequency response does not change linearly with respect to the applied load because of this mechanically composite structure. Therefore, the tape-based flexible sensor's response is more sensitive and more linear than the silicon-based sensor.

In conclusion, greater sensitivity and smaller nonlinearity error were achieved with the tape-based flexible sensor as compared to the silicon-based sensor. This is largely due to the greater compliance of the vacuum tape. In addition, the flexible tape sensor does not require the use of external epoxy between test material and vacuum tape substrate, which also contributes to its relatively greater sensitivity. The data indicate an improved sensitivity of $0.292 \mathrm{MHz} / \mathrm{kgf}$, or $13.83 \times 10^{-3} \mathrm{MHz} /$ microstrain, from the tape-based flexible sensor while the silicon-based sensor demonstrated a sensitivity of $0.0487 \mathrm{MHz} / \mathrm{kgf}$ or $2.303 \times 10^{-3} \mathrm{MHz} /$ microstrain. In addition, reduced nonlinearity errors of less than $80 \mathrm{mi}-$ crostrain (less than 3\%) in the tape-based flexible sensor was obtained as compared to nonlinearity errors less than 600 microstrain $(50 \%)$ that were calculated from the siliconbased sensor data.

This work is supported by Turkish National Academy of Sciences Distinguished Young Scientist Award (TÜBA GEBIP), European Science Foundation European Young Investigator Award (ESF-EURYI), a subcontract from NIH 5R01EB010035-02, and TÜBİTAK EEEAG Grant Nos. 105E066, 105E065, 104E114, 106E020, 107E088, and 107E297, and EU MOON Grant No. 021391.

${ }^{1}$ A. Ghali and R. Favre, Concrete Structures: Stresses and Deformations (E \& FN Spon, London, 1994).

${ }^{2}$ K. Stoffel, K. Klaue, and S. M. Perren, Injury 31, 37 (2000).

${ }^{3}$ R. Melik, N. K. Perkgoz, E. Unal, C. Puttlitz, and H. V. Demir, J. Micromech. Microeng. 18, 115017 (2008).

${ }^{4}$ R. Melik, E. Unal, N. K. Perkgoz, C. Puttlitz, and H. V. Demir, Appl. Phys. Lett. 95, 011106 (2009).

${ }^{5}$ R. Melik, E. Unal, N. K. Perkgoz, B. Santoni, D. Kamstock, C. Puttlitz, and H. V. Demir, "Nested metamaterials for wireless strain sensing," IEEE J. Sel. Top. Quantum Electron. (to be published), special issue on Metamaterials.

${ }^{6}$ J. B. Pendry, A. J. Holden, D. J. Robbins, and W. J. Stewart, J. Phys.: Condens. Matter 10, 4785 (1998).

${ }^{7}$ X. Wang, D.-H. Kwon, D. H. Werner, I.-C. Khoo, A. V. Kildishev, and V. M. Shalaev, Appl. Phys. Lett. 91, 143122 (2007).

${ }^{8}$ J. B. Pendry, Phys. Rev. Lett. 85, 3966 (2000).

${ }^{9}$ Z. W. Liu, N. Fang, T. J. Yen, and X. Zhang, Appl. Phys. Lett. 83, 5184 (2003).

${ }^{10}$ D. Schurig, J. J. Mock, B. J. Justice, S. A. Cummer, J. B. Pendry, A. F. Starr, and D. R. Smith, Science 314, 977 (2006).

${ }^{11}$ D. J. Quinn, "Microstructure, residual stress, and mechanical properties of thin film materials for a microfabricated solid oxide fuel cell," M.S. thesis, Massachusetts Institute of Technology, 2006.

${ }^{12}$ See EPAPS supplementary material at http://dx.doi.org/10.1063/ 1.3250175 for nonlinearity-errors of silicon-based sensor and tape-based flexible sensor in terms of percentage. 